\title{
Precision EW results from CMS
}

\section{Marco Cipriani* ${ }^{* \dagger}$ on behalf of the CMS Collaboration}

Sapienza Università di Roma \& INFN Romal

E-mail: marco.ciprianiecern.ch

Precision measurements of the properties of $\mathrm{W}$ and $\mathrm{Z}$ bosons at hadron colliders are a powerful tool to test the internal consistency of the Standard Model (SM) of particle physics. Theoretical predictions of their total production cross section, as well as differential distributions, are often available with next-to-leading order accuracy or beyond in both the strong and electroweak (EW) coupling constants with sub-percent precision.

The large amount of data collected at the CERN Large Hadron Collider (LHC) in proton-proton collisions at a center-of-mass energy of 8 and $13 \mathrm{TeV}$ provides an unprecedented opportunity to perform precision measurements of the EW sector of the SM with negligible statistical uncertainty. These measurements allow to test perturbative quantum chromodynamics and EW calculations, and are extremely valuable to set stringent constraints on the parton distribution functions. The deep understanding of the production of EW bosons at the LHC is a fundamental ingredient to perform precision measurements of the SM, and is also mandatory to discover new physics. Indeed, the largest source of irreducible background in many searches for new phenomena is represented by the production of undetected neutrinos from decays of $\mathrm{W}$ and $\mathrm{Z}$ bosons, and the typical experimental signature for a new physics signal is given by deviations from the precise SM prediction of kinematics distributions.

This paper describes some of the most recent EW precision measurements performed by the CMS Collaboration at the LHC. These measurements provide the theoretical community with precious information that will support the development of more accurate predictions. In addition, these results improve the current knowledge of the EW sector and will help chart the course for future measurements.

ALPS 2019 An Alpine LHC Physics Summit

April 22 - 27, 2019

Obergurgl, Austria

\footnotetext{
*Speaker.

${ }^{\dagger}$ The speaker would like to thank the conference organizers for their kind invitation.
} 


\section{Introduction}

The Standard Model (SM) of particle physics describes matter in terms of fundamental particles and their interactions mediated by vector bosons. The theory has been extremely successful in describing several natural phenomena, showing also an amazing predictive capability. For instance, the $\mathrm{W}$ and $\mathrm{Z}$ bosons were theorized about 20 years before their discovery at CERN in 1983. The production of $\mathrm{W}$ and $\mathrm{Z}$ bosons is one of the most prominent examples of hard scattering processes at hadron colliders, such as the Large Hadron Collider (LHC) at CERN. Measurements of the corresponding inclusive and differential cross sections provide stringent tests of quantum chromodynamics (QCD) and electroweak (EW) calculations, and parton distribution functions (PDFs). Theoretical calculations are often available with next-to-leading order (NLO) or higher accuracy in the QCD and EW couplings, leading to predictions with a precision at the level of few percent or better. The CMS experiment [1] at the LHC has performed many cross section measurements for vector bosons, either singly produced or in association with other SM particles. The range of measured cross sections span over several orders of magnitude, and a very good agreement with theory is observed at different center-of-mass energies. This extraordinary achievement is the result of a fruitful collaboration between theory and experiments.

The deep understanding of the production of EW bosons at the LHC is a fundamental ingredient for several SM precision measurements, such as the $\mathrm{W}$ boson mass [2]. Moreover, the precise knowledge of kinematic spectra for EW processes is also vital to probe new physics beyond the SM. Indeed, new phenomena are usually explained by postulating the existence of unknown particles that are weakly coupled to SM ones. These particles would not interact with the detectors, and the SM production of neutrinos from decays of $\mathrm{W}$ and $\mathrm{Z}$ bosons often represents the main source of irreducible background. Therefore, precision measurements are not only a valuable tool to test the internal consistency of the SM, but also a powerful handle to indirectly discover new physics through the observation of small deviations from the precise SM predictions.

This paper presents some of the latest precision EW measurements published by the CMS experiment using data collected at a center-of-mass energy of 8 or $13 \mathrm{TeV}$. They are carried out in the $\mathrm{W}$ and $\mathrm{Z}$ leptonic decay channel, which provides a clean experimental signature with one or more high-energy and isolated charged leptons in the final state. The production of a charged lepton allows to suppress the large background from QCD multi-jet production, which is copious at hadron colliders. Moreover, the lepton momentum is measured with high resolution and accuracy. In some cases, results are compared with predictions from many Monte Carlo (MC) generators, which differ in their approach to model the emission of additional jets. Depending on the generator and the considered phase space, different levels of agreement are observed. This is a valuable source of information, as it can suggest the need for missing higher order corrections or a better accuracy in the modeling of non-perturbative effects.

\section{Measurements at $8 \mathrm{TeV}$}

The weak mixing angle $\sin ^{2} \theta_{W}$ is one of the fundamental parameters of the SM. At leading order, it is related to the masses of the vector bosons by $\sin ^{2} \theta_{W}=1-m_{W}^{2} / m_{Z}^{2}$, but higher order EW corrections can be simply absorbed into an effective weak mixing angle $\sin ^{2} \theta_{\text {eff }}^{\ell}$ [3]. The differen- 
tial cross section for the Drell-Yan dilepton production through $q \bar{q} \rightarrow Z / \gamma^{*} \rightarrow \ell^{+} \ell^{-}$can be written as $d \sigma / d\left(\cos \theta^{*}\right) \propto 1+\cos ^{2} \theta^{*}+A_{4} \cos \theta^{*}$, where $\theta^{*}$ is the angle of the $\ell^{-}$in the Collins-Soper frame. The $\cos \theta^{*}$ term is due to interference between the vector and axial-vector contributions, and results in a forward-backward asymmetry $\left(A_{F B}\right)$ that is sensitive to $\sin ^{2} \theta_{e f f}^{\ell}$. The size of $A_{F B}$ depends on the flavor of the initial-state quarks, thus on the PDFs. In addition, its definition assumes that the lepton pair is Lorentz-boosted along the direction of the quark, implying further dependence on the PDFs due to large-x initial-state $\bar{q}$.

The CMS measurement of $\sin ^{2} \theta_{\text {eff }}^{\ell}$ was performed on the full dataset at $8 \mathrm{TeV}$ [3]. It is based on a template fit to the observed $A_{F B}$ as a function of the dilepton mass and rapidity $(y)$. The nominal signal templates, obtained for different hypotheses of $\sin ^{2} \theta_{e f f}^{\ell}$, are generated using POWHEG at NLO in QCD and LO in EW with NLO NNPDF3.0 PDFs. The observed and fitted $A_{F B}\left(m_{\ell \ell}, y\right)$ in the muon channel is shown in Fig. 1a. It should be noted that $A_{F B}$ is maximal at high $|y|$. This corresponds to the forward region of the detector, where the measurement is more sensitive to PDFs.

The combination of the $e e$ and $\mu \mu$ channels yields $\sin ^{2} \theta_{\text {eff }}^{\ell}=0.23101 \pm 0.00036$ (stat) \pm 0.00018 (syst) \pm 0.00016 (theory) \pm 0.00031 (PDF). The dominant uncertainty is the statistical one, followed by the PDF systematic uncertainty. Therefore, the precision of this measurement is expected to improve by using the larger dataset already available at $13 \mathrm{TeV}$. Moreover, ther PDF uncertainty could be reduced by extending the analysis acceptance to larger $y$ values.

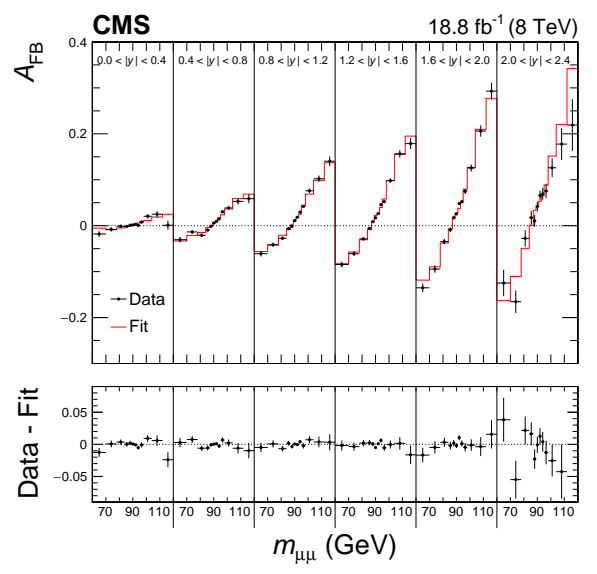

(a) $A_{F B}$ in $Z \rightarrow \mu \mu$ events

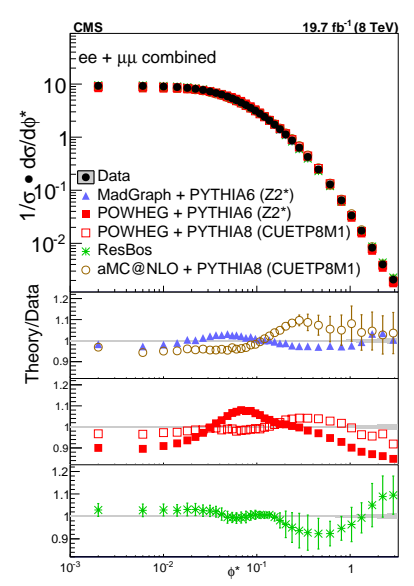

(b) Z boson $\mathrm{d} \sigma / \mathrm{d} \phi^{*}$

Figure 1: Left: $A_{F B}$ measured in $Z \rightarrow \mu \mu$ events as a function of the mass and rapidity of the lepton pair. Right: Z-boson differential cross section as a function of $\phi^{*}$ normalized to the total one, from the combination of the electron and muon channels. Taken from $[3,4]$

At the $\mathrm{LHC}, \mathrm{W}$ and $\mathrm{Z}$ bosons are produced with non-zero momentum transverse to the beam axis, due to the intrinsic transverse momentum $\left(p_{T}\right)$ of the initial-state partons inside the protons and the initial-state radiation of gluons and quarks. The $p_{T}$ distribution of the boson can be computed with fixed-order perturbative QCD calculations for $p_{T} \gtrsim m_{Z / W}$. However, fixed-order calculations become unreliable for $p_{T} \lesssim 20 \mathrm{GeV}$ due to large logarithmic terms, which need to be treated with resummation techniques. Therefore, direct measurements targeting the low- $p_{T}$ regime are precious. Events with a $\mathrm{Z}$ boson decaying into a pair of charged leptons are more suited for this 
measurement, as the final state can be fully reconstructed with high resolution.

The experimental uncertainty on $p_{T}^{Z}$ is dominated by the uncertainties in the magnitude of the $p_{T}$ of the leptons. However, thanks to the excellent spatial resolution of the CMS tracker, the angles subtended by the leptons can be measured with much higher precision. This feature was exploited by CMS to measure the Z-boson differential cross section ${ }^{1}$ as a function of $\phi^{*}$, using $19.7 \mathrm{fb}^{-1}$ [4]. The $\phi^{*}$ variable is correlated with $p_{T}^{Z}$ by the approximate relation $\phi^{*} \approx p_{T}^{Z} / m_{\ell \ell}$, but depends only on angular variables and allows to investigate the low- $p_{T}$ region with higher resolution. The measured cross section, normalized to the total one, is shown in Fig. 1b. The dominant uncertainties stem from the finite size of the event samples in both data and MC. The observed distribution is compared with predictions from different combinations of hard scattering and parton shower generators. Better models of the hard-scattering process, as provided by MADGRAPH + PYTHIA 6, lead to an improved agreement with data. At the same time, the comparison with POWHEG interfaced with two different versions of PYTHIA demonstrates the importance of the underlying event model and hadronization tune to yield the most accurate description of data.

\section{Measurements at $13 \mathrm{TeV}$}

The high center-of-mass energy of proton collisions at the LHC facilitates the production of vector bosons with one or more high- $p_{T}$ hadronic jets. The leading production mechanism is driven by QCD processes, where the vector boson is directly emitted from the colliding quarks. An alternative mode is given by EW production through vector boson fusion (VBF). The corresponding cross section is a factor $10^{-4}$ lower than the QCD process, but is characterized by a distinctive signature with two forward jets with large combined mass and angular separation. The study of the VBF mechanism is important for measurements of the Higgs boson, which has a similar production mode. In addition, some theories beyond the SM predict anomalous triple gauge couplings (ATGCs) induced by higher-dimension operator, related to the WWZ vertex. Therefore, the measurement of the coupling strengths in the EW production potentially probes new physics at mass scales not directly accessible at the LHC.

CMS measured the cross section for the EW production of $\mathrm{W}$ and $\mathrm{Z}$ bosons using $35.9 \mathrm{fb}^{-1}$ of data collected at $13 \mathrm{TeV}[5,6]$. Multivariate analysis techniques were used, which exploit the distinctive topology of the EW production mechanism to enhance the signal purity over the large background arising from the QCD production. The measured cross sections from the combination of the muon and electron decay channels are $\sigma_{E W}(W j j)=6.23 \pm 0.12$ (stat) \pm 0.61 (syst) pb and $\sigma_{E W}(Z j j)=534 \pm 20$ (stat) \pm 57 (syst) fb for $\mathrm{W}$ and $\mathrm{Z}$ bosons, respectively. The total uncertainty is dominated by the experimental systematic component due to the jet energy scale and resolution.

A search for ATGCs was also performed, looking for an enhancement of the cross section at high values of the $p_{T}$ of the lepton pair or the charged lepton for $\mathrm{Z}$ or $\mathrm{W}$ bosons, respectively. No sign of new physics was found, but the combination of the CMS searches in the $\mathrm{Z}$ and $\mathrm{W}$ channel allowed to set the most stringent limits to date on the new physics coupling $c_{W W W}$. Figure 2a shows the limits on the two couplings $c_{W}$ and $c_{W W W}$. The EW production mode is also an interesting channel to study the jet activity between forward jets. This allows to improve the modeling of

\footnotetext{
${ }^{1} \mathrm{~A}$ new CMS measurement with $35.9 \mathrm{fb}^{-1}$ at $13 \mathrm{TeV}$ became public in May 2019: http://cds.cern.ch/record/2675022
} 
additional parton radiation, and is important for signal selection and the vetoing of background events in Higgs boson analyses and searches for new physics.

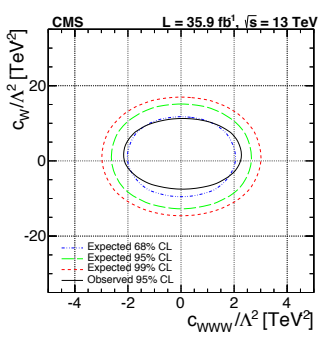

(a) Limits on ATGCs

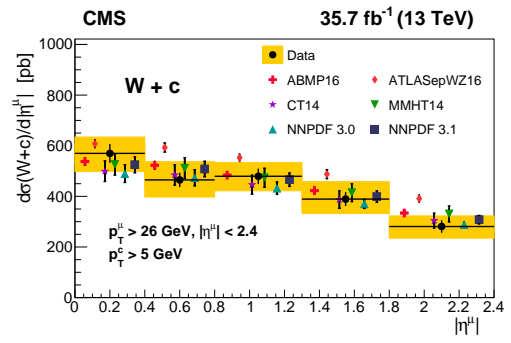

(b) $\mathrm{d} \sigma(W+c) / \mathrm{d}\left|\eta^{\mu}\right|$

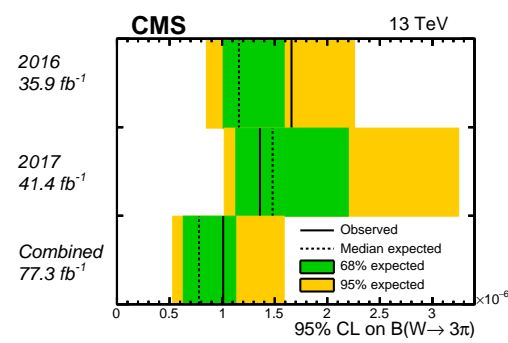

(c) $B(W \rightarrow 3 \pi)$

Figure 2: Left: limits on ATGCs from the combination of the $\mathrm{W}$ and $\mathrm{Z}$ analysis in the EW production mode. Middle: $\mathrm{W}+\mathrm{c}$ differential cross section as a function of the $|\eta|$ of the muon from the $\mathrm{W}$ decay. Right: Upper limits on the branching ratio for the decay of a $\mathrm{W}$ boson into 3 pions. Taken from $[5,7,8]$

The interpretation of results obtained in proton-proton collisions at the LHC relies on the knowledge of the PDFs of the proton. The production of vector bosons, inclusive or in association with quarks, carries useful information about the flavor composition of the light quark sea inside the proton. In particular, the associated production of a $\mathrm{W}$ boson with a charm quark [7] is sensitive to the strange quark PDF through the leading order process $g+s \rightarrow W+c$. It also provides an important cross-check of the results from global PDF fits to deep inelastic scattering (DIS) data.

Signal events are characterized by a $\mathrm{W}$ boson and a charm quark with opposite sign (OS). The $\mathrm{W}$ boson is identified through its decay into a muon, while the charm quark is tagged through the reconstruction of the decay chain of charmed hadrons in the process $c \rightarrow D^{*}(2010)^{ \pm} \rightarrow D^{0}(1865)+$ $\pi_{\text {slow }}^{ \pm} \rightarrow K^{\mp}+\pi^{ \pm}+\pi_{\text {slow }}^{ \pm}$, where $\pi_{\text {slow }}^{ \pm}$denotes a charged pion with low momentum due to the small mass difference between the $D^{*}$ and $D^{o}$ mesons. The selection of signal events benefits from the excellent performance of the CMS tracker in the reconstruction of tracks and location of secondary vertices from the decays of charmed hadrons. The $D^{*}$ candidates are identified using the mass difference method through the peak in the $\Delta m\left(D^{*}, D^{0}\right)$ distribution. The main background consists of events with $W+c \bar{c}$ and $W+b \bar{b}$ : these processes produce an equal amount of charm quarks with opposite and same sign (SS) as the W boson. Therefore, the contribution of OS background events in the signal region can be removed by subtracting the number of SS events.

The measured $W+c$ cross section, differential in the muon pseudorapidity, is shown in Fig. 2b. Results are compared with QCD predictions calculated with MCFM at NLO using different NLO PDF sets. This measurement was used in an NLO QCD analysis with inclusive DIS measurements and earlier results from CMS, resulting in better constraints of the s-quark PDF.

The last analysis described in this paper is the first search for the rare decay of the $\mathrm{W}$ boson into 3 charged pions [8]. There are no theoretical predictions for this branching ratio, but it is expected to be between $10^{-8}$ and $10^{-5}$. If observed, this channel would provide a new way to measure the $\mathrm{W}$ boson mass, as the final state consists solely of visible tracks from the pions. The analyzed dataset corresponds to $77.3 \mathrm{fb}^{-1}$ collected by CMS during 2016 and 2017 . The analysis relies on an innovative use of algorithms originally designed to trigger on and identify hadronically decaying $\tau$ leptons. The signal is extracted through a binned maximum likelihood fit to the invariant mass distribution of the 3 pions. The sensitivity of the analysis is limited by the finite sample of 
selected events. The leading systematic uncertainty stems from the pion identification efficiency and amounts to 5\% per pion. No signal was observed above the background expectation, and an upper limit on the branching ratio was set, yielding $B R(W \rightarrow 3 \pi)<1.01 \times 10^{-6}$, as shown in Fig 2c. This limit already restricts the range of possible branching ratios for this rare process, and it will definitely improve with more data.

\section{Summary}

Some of the latest precision EW measurements performed with the CMS detector have been presented. These measurements provide stringent tests of perturbative QCD and EW calculations, as well as constraints on the PDFs of the proton. They also play a key role in probing new physics through deviations from the precise SM predictions.

The uncertainty on the measured quantities is generally dominated by either the statistical uncertainty in data and MC samples, or theoretical systematic uncertainties. The latter stem from the modeling of the boson production mechanism. Theoretical uncertainties can be constrained in the future through dedicated ancillary measurements and improved theoretical calculations.

A large amount of data has been collected so far thanks to the excellent performance of both the LHC and the CMS detector, allowing to probe the SM with unprecedented precision and explore rare processes. More data will become available in the coming years, leading to further improvements through the usage of new analysis techniques and the developments of new measurements. The collaboration between experiments and theory is vital to fully exploit this benefit.

\section{References}

[1] S. Chatrchyan et al. [CMS Collaboration], The CMS experiment at the CERN LHC, JINST 3, S08004 (2008).

[2] M. Aaboud et al. [ATLAS Collaboration], Measurement of the W-boson mass in pp collisions at $\sqrt{s}=$ $7 \mathrm{TeV}$ with the ATLAS detector, Eur.Phys. J. C 70, (2018) 110.

[3] A. M. Sirunyan et al. [CMS Collaboration], Measurement of the weak mixing angle using the forward-backward asymmetry of Drell-Yan events in pp collisions at 8 TeV, Eur. Phys. J. C 78, (2018) 701.

[4] A. M. Sirunyan et al. [CMS Collaboration], Measurement of differential cross sections in the kinematic angular variable $\phi^{*}$ for inclusive $Z$ boson production in pp collisions at $\sqrt{s}=8 \mathrm{TeV}, \mathrm{JHEP}$ 03, (2018) 172.

[5] CMS Collaboration, Measurement of electroweak production of a $W$ boson in association with two jets in proton-proton collisions at $\sqrt{s}=13 \mathrm{TeV}$, CMS-SMP-17-011, Submitted to Eur. Phys. J. C.; [arXiv:1903.04040v1 [hep-ex]]

[6] A. M. Sirunyan et al. [CMS Collaboration], Electroweak production of two jets in association with a $Z$ boson in proton-proton collisions at $\sqrt{s}=13 \mathrm{TeV}$, Eur. Phys. J. C 78, (2018) 589.

[7] A. M. Sirunyan et al. [CMS Collaboration], Measurement of associated production of a W boson and a charm quark in proton-proton collisions at $\sqrt{s}=13 \mathrm{TeV}$, Eur. Phys. J. C 79, (2019) 269.

[8] A. M. Sirunyan et al. [CMS Collaboration], Search for W boson decays to three charged pions, Phys. Rev. Lett. 122, (2019) 151802. 